\title{
Dynamic visible interferometric measurement of thermal fields around living biological objects
}

\author{
Katherine Creath $^{* a, b, c}$, Gary E. Schwartz ${ }^{\mathrm{a}, \mathrm{c}}$ \\ ${ }^{a}$ Biofield Optics, LLC, 2247 E. La Mirada St., Tucson, AZ, USA 85719; \\ ${ }^{\mathrm{b}}$ Optical Sciences Center, University of Arizona, 1630 E. University Blvd, \\ Tucson, AZ, USA 85721-0094; \\ ${ }^{\mathrm{c}}$ Center for Frontier Medicine in Biofield Science, University of Arizona, \\ 1601 N. Tucson Blvd., Su.17, Tucson, AZ, USA 85719
}

\begin{abstract}
Dynamic interferometry is a highly sensitive means of obtaining phase information that can determine phase at rates of a few measurements per second. The sensitivity of these phase-measurement instruments is on the order of thousandths of a wavelength at visible wavelengths enabling the measurement of small temperature changes and thermal fields surrounding living biological objects. Temperature differences are clearly noticeable using a visible wavelength source because of subtle changes in the refractive index of air due to thermal variations between an object and the ambient room temperature. Living objects can also easily be measured over a period of time to monitor changes as a function of time. This technique has many promising applications in biological and medical sciences for studying thermal fields around living objects. In this paper we compare differences in thermal fields measured with dynamic phase-measuring interferometry surrounding room temperature and body temperature inanimate objects as well as living biological objects at data rates of many measurements per second.
\end{abstract}

Keywords: dynamic interferometry, phase measurement, biomedical optics, optical instrumentation

\section{INTRODUCTION}

Biofields are fields surrounding living biological objects. ${ }^{1}$ They can have thermal, photonic, magnetic and electromagnetic components. ${ }^{2}$ The research presented in this paper has grown out of a desire to develop instrumentation to measure fields around living objects to support research in complementary and alternative medicine (CAM). ${ }^{3}$ In particular, our interest is to develop techniques to detect subtle changes in biofields that can be linked to state of health in humans.

For this work we are using dynamic interferometry as a means of looking at thermal fluctuations around living biological objects. Since interferograms are sensitive to subtle changes in refractive index between the object and reference beams, dynamic interferometry provides real-time sensitive measurements that can be tracked over time. The work presented in this paper shows a proof of concept of this technique for real-time measurements of thermal biofields close to and surrounding biological objects.

\section{DYNAMIC INTERFEROMETER}

Dynamic interferometry is a highly sensitive means of obtaining phase information that can take phase data at rates of multiple frames per second. ${ }^{4,5}$ Dynamic interferometry techniques enable taking all necessary interferometric data simultaneously to determine phase in a single snapshot. ${ }^{6-8}$ These instruments have been designed with the purpose of measuring phase data in the presence of vibration and air turbulence. ${ }^{9,}{ }^{10}$ Interferograms can be captured in "real" time. Turbulence in the area near an object can be "frozen." Flows and vibrational motion can be followed. ${ }^{11}$ Quantitative phase maps can be obtained over a time series to create movies ("burst" mode) of the optical path (OPD) variations over time due to a living biological object.

*kcreath@,ieee.org; phone 1520 882-2950; fax 1520 882-6976 
The specific type of dynamic interferometry used for this study is a spatial multichannel phase-measurement technique. ${ }^{12}$ Multiple duplicate interferograms are generated with different relative phase shifts between the object and reference beams. These interferograms are then recorded using either a number of separate cameras or by multiplexing all of the interferograms onto a single camera. These interferometers are well suited for studying objects that are not vibrationally isolated or that are dynamically moving. They are able to "freeze" the motion of an object to obtain phase and surface height information as a function of time. Commercial systems utilizing this type of phase measurement are manufactured by 4D Technology Corporation, ${ }^{13}$ Optical Device Engineering Corporation, ${ }^{14}$ and ADE Phase Shift. ${ }^{15}$ The system used for this study was a PhaseCam ${ }^{\mathrm{TM}}$ from 4D Technology. ${ }^{16,} 17$

A schematic of the 4D Technology PhaseCam is shown in Fig. 1 below. A single mode polarized HeNe laser beam is expanded and collimated to provide illumination coupled in by a polarization beam splitter. Quarter-wave plates are used to set orthogonal polarizations for the object and reference beams. The object beam is further expanded, collimated and directed at a return mirror. The cavity between the collimating lens and the return mirror is where the objects were placed for this study. When the object and reference beams are recombined inside the interferometer, the polarizations are kept orthogonal. The combined beams pass through optical transfer elements providing imaging of the return mirror onto the high-resolution camera.

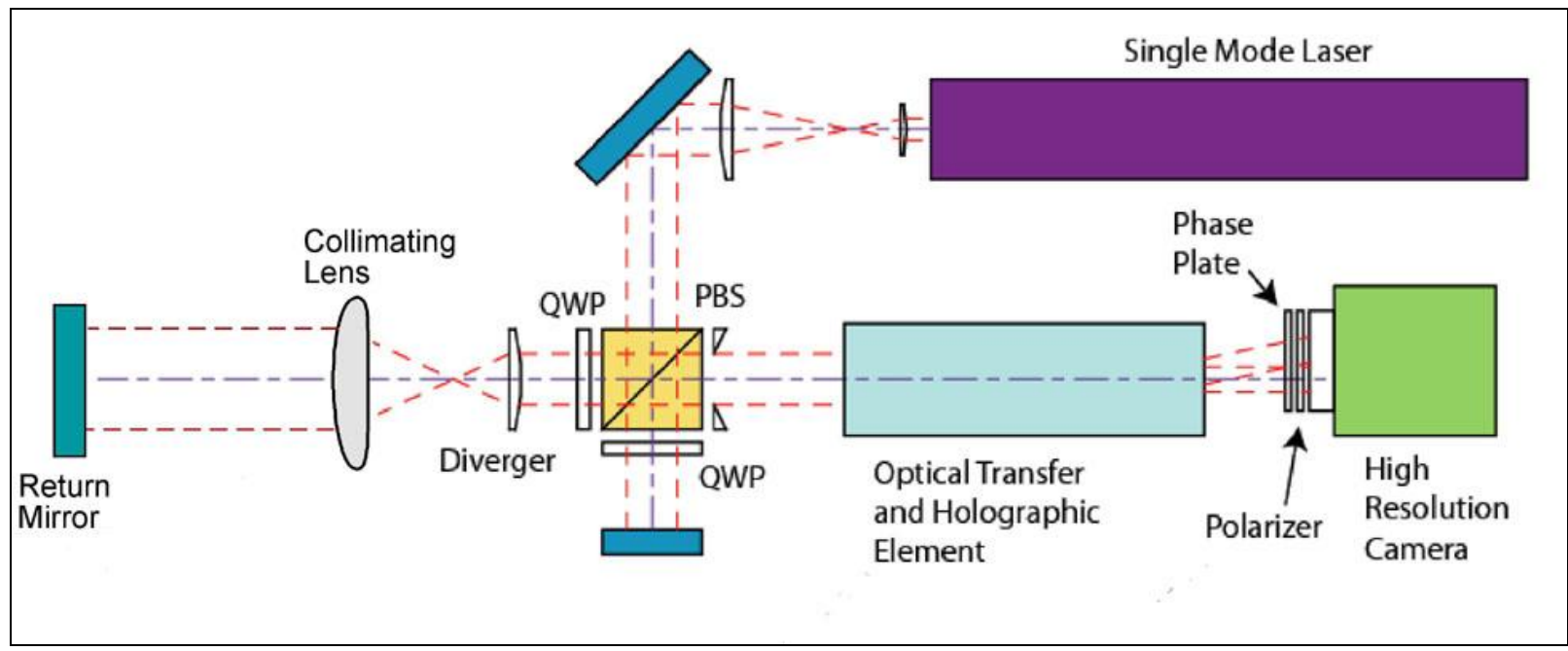

Fig. 1. Schematic of dynamic interferometer system used for this study. The object under study is between the collimating lens and the return mirror. (Courtesy of 4D Technology Corporation).

A holographic optical element duplicates the beams into four copies of the interference pattern that are mapped onto four quadrants of the camera (see Fig. 2). A phase plate consisting of polarization components is placed in front of the camera to provide a different relative phase shift between the object and reference beams in each of the four quadrants of the image plane. Phase values are determined modulo $2 \pi$ at each point in the phase map using the standard 4 -frame algorithm (see Fig. 2). ${ }^{18}$

This calculation does not yield absolute phase differences in the object cavity. The arctangent phase calculation provides modulo $2 \pi$ data requiring that phase values be unwrapped to determine a phase map of the relative phase difference between the object and reference beams. ${ }^{19}$ If data frames are tracked in time, and the phase values do not jump by more than a half of a fringe between frames of data, it is in theory possible to track the relative phase differences dynamically (also known as unwrapping in time). The version of the software used for this study unfortunately did not yet have the capability of unwrapping the phase in time.

Because the interferograms are multiplexed onto different quadrants of the image plane, care needs to be taken to determine pixel overlap of all the interferograms, to remove spatial optical distortion and to balance the gain values between interferograms. In practice, when measurements are taken, a reference file is generated from an average of a large number of frames of data with an empty cavity and subtracted from subsequent measurements to provide a null. 


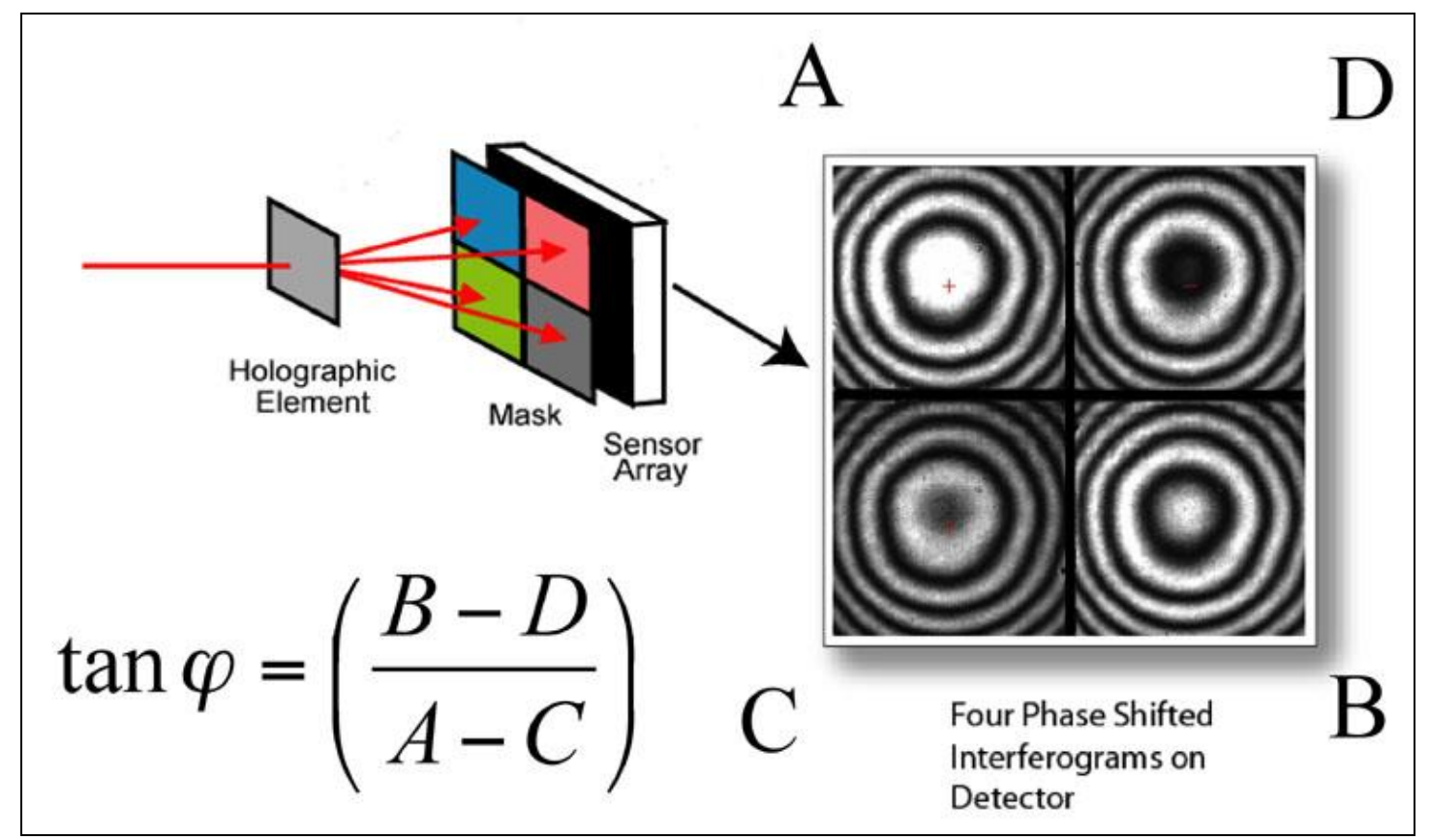

Fig. 2. Schematic showing the creation and encoding of 4 phase shifted interferograms onto the CCD camera. (Courtesy of 4D Technology Corporation).

Dynamic variations in the object cavity can be monitored by taking a "burst" of data comprised of a user-selectable number of data frames with a fixed time delay between frames. This lets us follow dynamic motion as a function of time. The sensitivity of phase-measurements taken with this instrument is on the order of thousandths of a wavelength at the visible $\mathrm{HeNe}$ wavelength $(633 \mathrm{~nm})$. This sensitivity enables the measurement of small temperature changes and thermal fields surrounding living biological objects.

\section{HYPOTHESES AND BACKGROUND}

The human body emits thermal radiation as well as light as part of basic metabolic processes. Biophoton emission from hands and other body parts have been measured with photomultiplier tubes by many researchers. ${ }^{20,}{ }^{21}$ The results of Cohen and Popp ${ }^{20}$ show that the emission varies depending upon state of health, position on the body, time of day, and time of the year. However, because of the nature of PMTs, images cannot be obtained to generate detailed emission maps. Recently, we have demonstrated that detailed images of biophoton emission can be obtained utilizing cooled, low-noise CCD cameras with exposure times on the order of minutes. ${ }^{22}$ Figure 3 shows images of the first author's hands taken by the second author using a cooled, highly sensitive silicon CCD camera with 10-minute exposures in total darkness. The Princeton Instruments VersArray 1300B camera was hardware binned providing 67x65 pixels with each pixel having a $400 \mu \mathrm{m}$ square sensing area. Note that the fingertips emit more biophotons than say the back of the hands, and the palms of the hands are in between. These images show great detail, but they do not enable us to look at dynamic changes on shorter time scales.

For the study presented in this paper we focused on looking at the difference between room temperature and body temperature objects and compared these to a human finger. The human body dynamically emits thermal energy. This thermal energy relates to metabolic processes. We hypothesize that the thermal emission from the human body is dynamic and cycles with time constants related to blood flow and respiration. These cycles create small bursts of thermal energy that creates convection and air currents. We have termed these subtle air currents generated by the human body "microbreezes". The thermal variations of these microbreezes modulate the refractive index of the air path. Because dynamic interferometry can measure subtle changes in refractive index and thereby measure air currents and microbreezes, we hypothesize that this technique will enable us to visualize the thermal aura around human body parts such as a finger tip, and furthermore that we will be able to quantify the relative variations over time. 


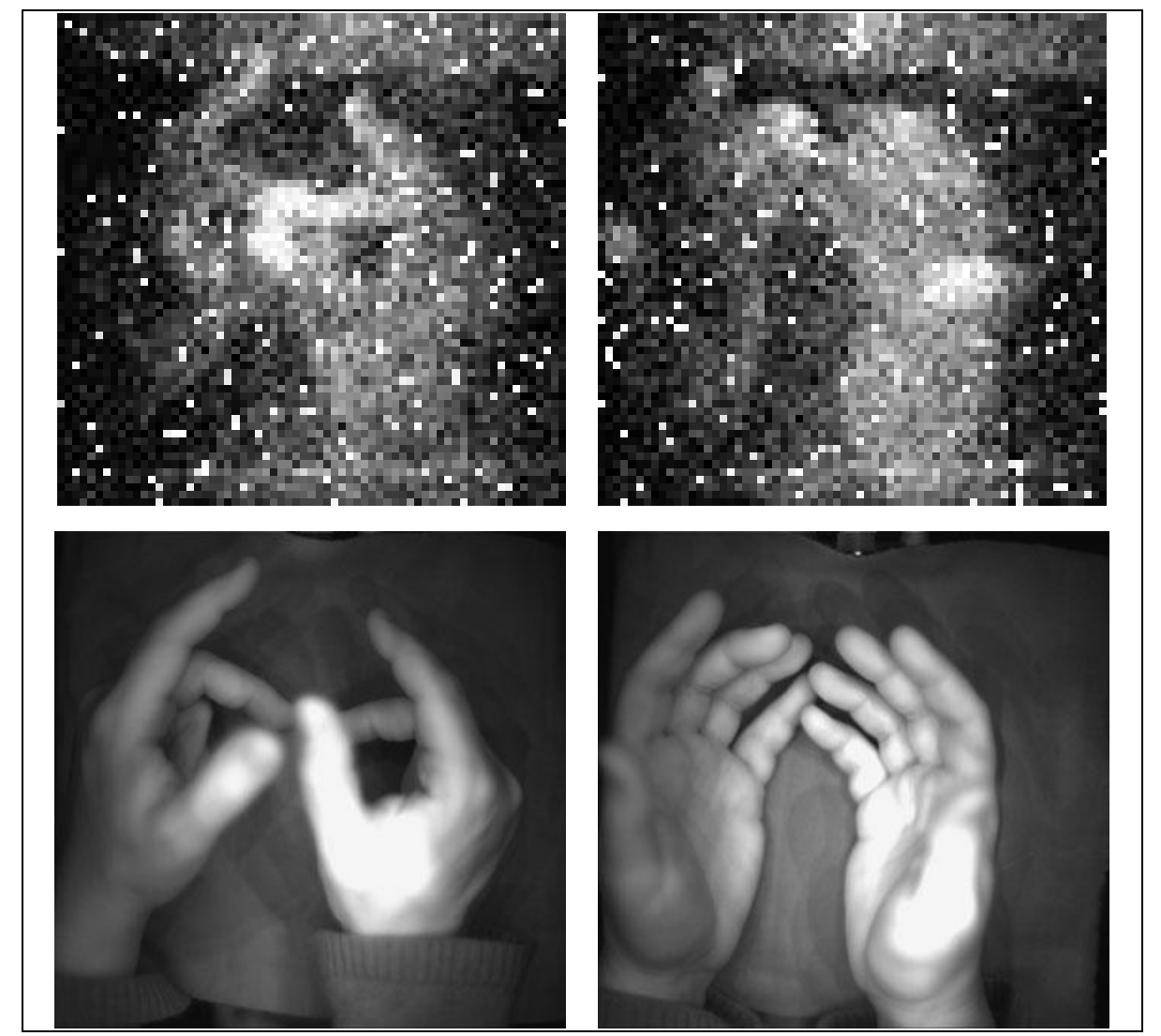

Fig. 3. Top images are 10-minute exposures taken in total darkness using 20x20 binning with a Princeton Instruments VersArray 1300B camera cooled to $-100^{\circ} \mathrm{C}$. These images represent biophoton emission (biological chemiluminescence) generated within the body as part of normal biological metabolic processes. Bottom images are 10ms exposures taken with white-light illumination.

\section{METHODOLOGY AND RESULTS}

The dynamic interferometer was set up as shown in Fig. 4. The object cavity between the collimating lens and the return mirror was enclosed with a cardboard tube except for an approximately $3 \mathrm{~cm}$ space to place the object in the beam. This limited ambient air currents from affecting the measurements as much as possible.

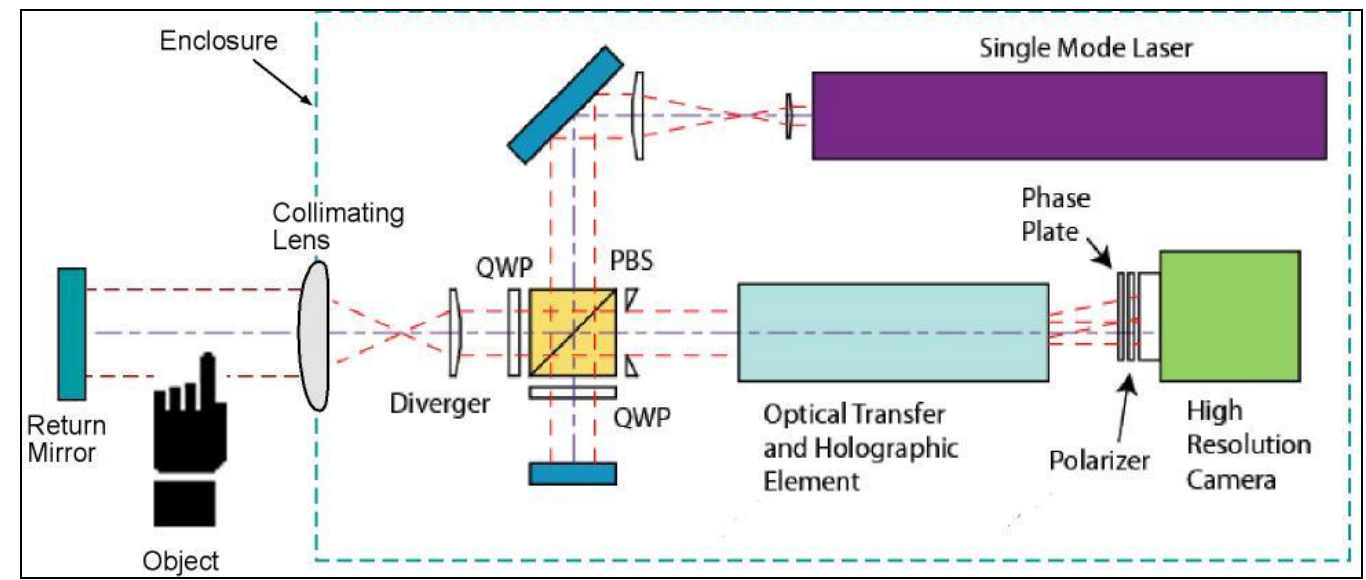

Fig. 4. Experimental setup. All beam paths are enclosed except for $3 \mathrm{~cm}$ in the object beam for placement of object. 
A reference data set was taken with no object present that was the average of 30 single measurements taken in a single burst. The phase maps were evaluated and averaged after all the data were taken. The reference data set was subtracted from all subsequent measurements to remove variations across the field due to the interferometer itself and create a null cavity when no object was present. Figure 5 A shows the empty object cavity scaled from -0.05 to +0.05 waves OPD (optical path difference between object and reference beams). Bright areas (yellow) are warmer than dark areas (dark blue). The phase maps are presented and scaled with a zero mean. Variations in this phase map are due to subtle air currents. Figure 5B shows a blast from a can of canned air. Note that the turbulence is easily frozen in time and that the canned air is cooler than the background air. Figure 5C shows the effect of a candle flame below the object beam. The area heated by the candle flame is obviously brighter than the darker ambient air temperature. These images are scaled from maximum to minimum with a zero mean. Because they are relative measurements we cannot get absolute temperature information. We can, however, determine relative changes in refractive index due to temperature variations.

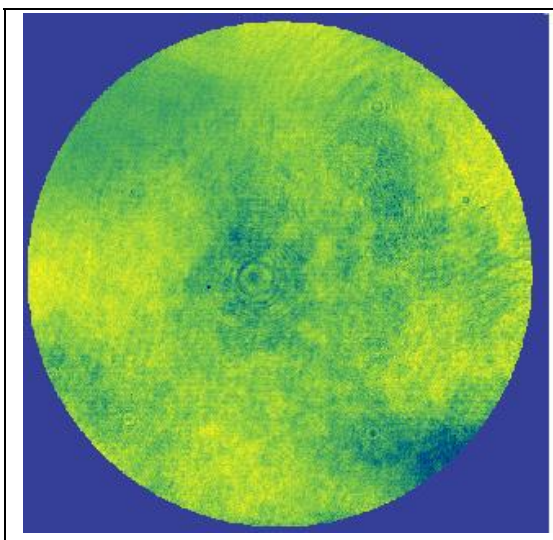

(A)

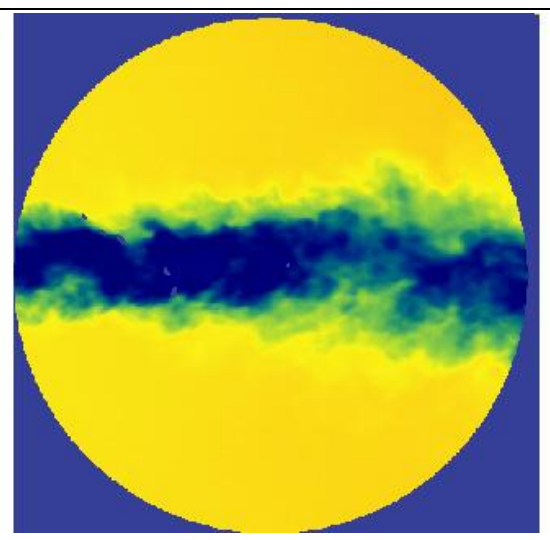

(B)

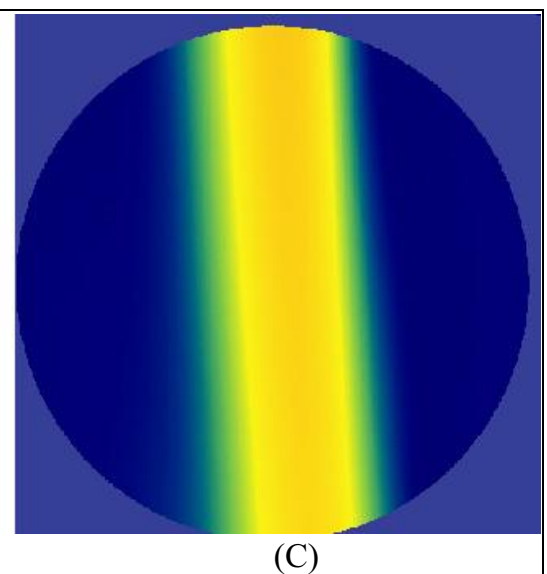

(C)

Fig. 5. (A) Empty object cavity. (B) Blast of canned air. (C) Candle flame. Bright (yellow) is warm and dark (blue) is cool.

The next set of measurements compares objects at different temperatures as well as an inanimate object to a living object at the same temperature. Figure $6 \mathrm{~A}$ shows a screwdriver handle approximately $2 \mathrm{~cm}$ across at room temperature. These images are scaled from -0.05 to +0.05 waves OPD with a zero mean to compare with Fig. 5A. The presence of the room temperature screwdriver handle does not appear to thermally affect the air path at all. However, when the screwdriver handle is warmed up to body temperature and placed in the beam, there is obviously a thermal gradient around it (Fig. 6B). Figure 6C shows a finger of the second author placed in the beam. Note that the thermal gradient around the finger is similar to that around the body temperature screwdriver handle. The differences between these two objects are mainly in the surrounding "halo".

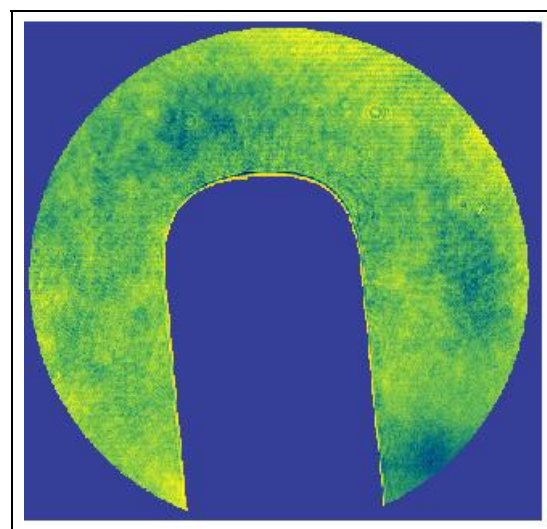

(A)

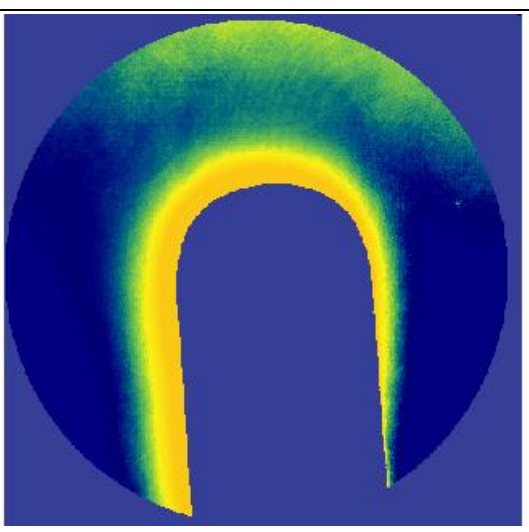

(B)

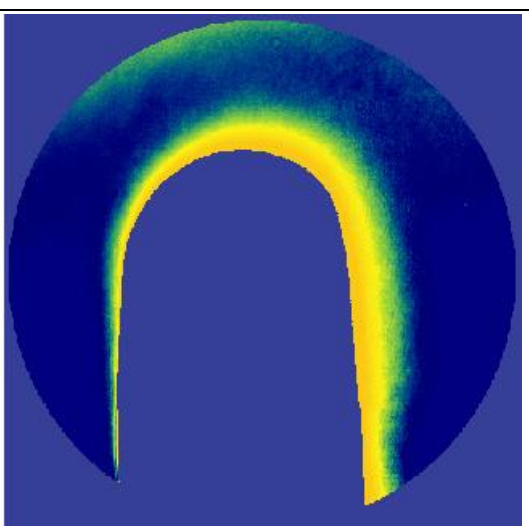

(C)

Fig. 6. (A) Room temperature screwdriver handle. (B) Body temperature screwdriver handle. (C) Human finger. All phase maps are scaled from +0.050 to -0.050 waves for comparison. 
To observe these objects dynamically, movies were recorded of the interference patterns and then phase maps were processed after the desired number of data frames was taken. The only limit to the number of data frames that can be taken in a single "burst" is limited by the space to store the interferogram images. Figure 7 shows 10 consecutive phase maps taken of the body temperature screwdriver handle. Each exposure was a standard video frame. The ten frames of Fig. 7 represent less than a second of total elapsed time. For each phase map, the software displays the data as zero mean and the phase scale is set from -0.10 to +0.10 waves OPD. Note that there is very little difference as a function of time in the field around the warm screwdriver handle.

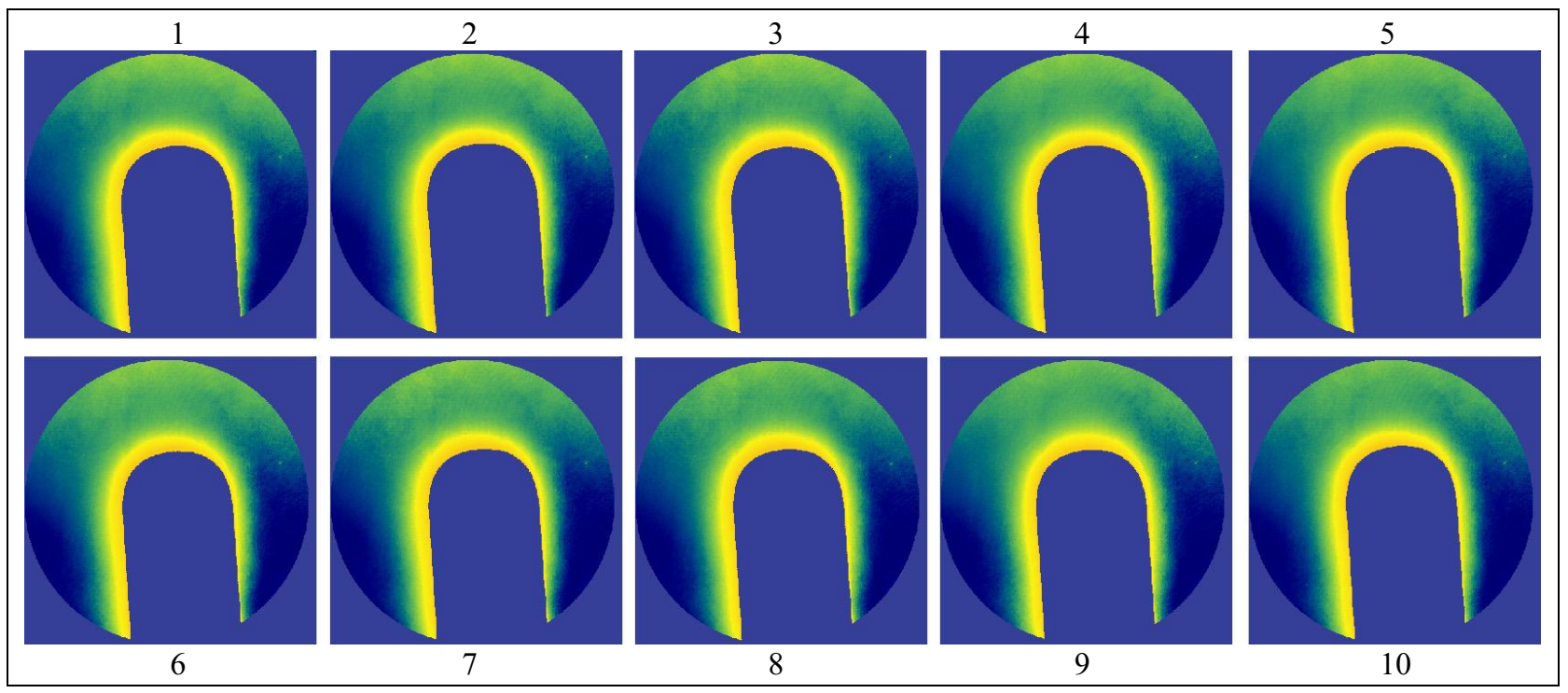

Fig. 7. Ten consecutive exposures of a body temperature screwdriver handle. All phase maps are zero mean and colors are scaled from +0.10 to -0.10 waves.

Figure 8 shows 10 consecutive phase maps taken by the first author of the second author's finger. Exposure times and time delays were set exactly the same as for the body temperature screwdriver handle of Fig. 7. Again each phase map is zero mean and scaled from -0.10 to +0.10 waves OPD. Note that there are variations in the field around the finger that change with time that are not noticeable in Fig. 7.
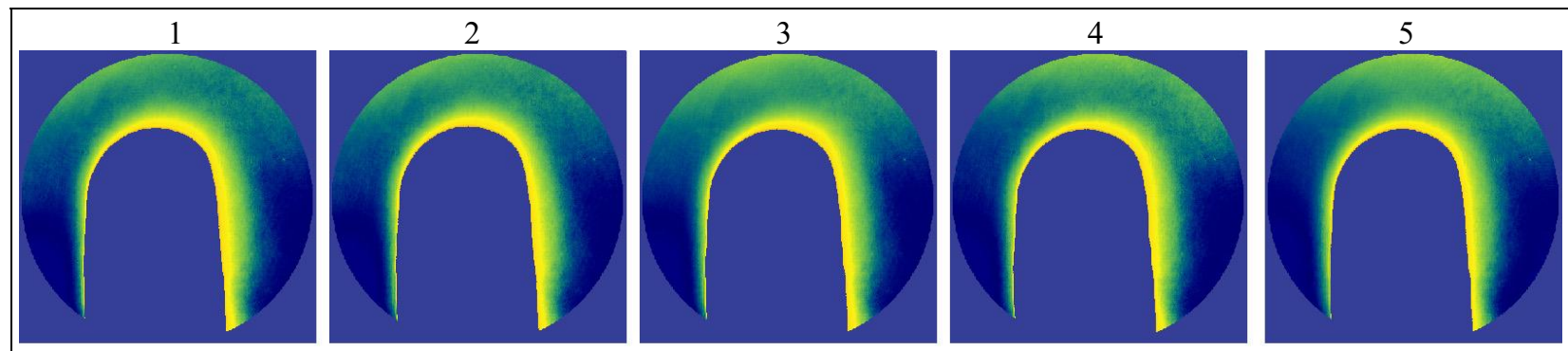

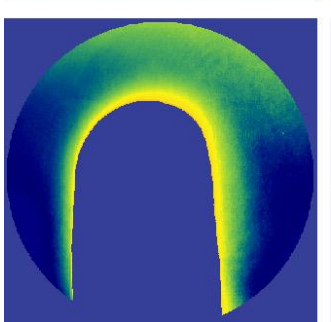

6

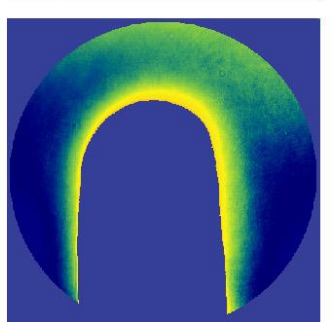

7

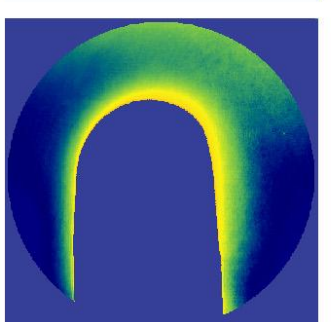

8

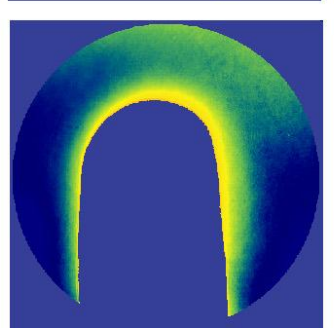

9

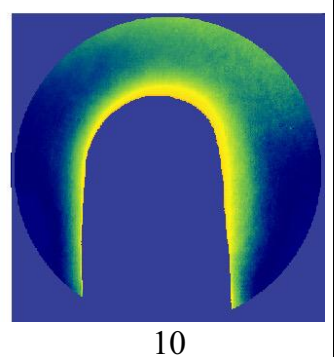

Fig. 8. Ten consecutive exposures of a human finger. All phase maps are zero mean and colors are scaled from +0.10 to -0.10 waves. 
The most noticeable variations are near the tip of the finger. When watched as a movie, these frames show motion of the air currents around the tip of the finger that corroborate our hypotheses of thermally emitted microbreezes. This dynamic motion is not noticeable around the end of the screwdriver handle when Fig. 7 is observed in movie mode. Another difference is the dynamic changes in the thermal gradient along the right side of the finger. This halo expands and contracts as a function of time. Again, this type of motion is not visible around the body temperature screwdriver handle.

\section{DISCUSSION}

The sensitivity of these phase measurements shows that our eye can easily discern 0.01 waves difference in OPD. Calculations can further extend the repeatability to have a sensitivity of around 0.001 waves. The refractive index of air is roughly 1.003 and is dependent upon temperature, pressure, humidity, $\mathrm{CO}_{2}$ quantity and wavelength. These dependencies have been studied extensively for accurate distance measurements using light. ${ }^{23}$ Operating with an interferometric measurement sensitivity on the order of 0.001 waves, variations of 1 part in $10^{4}$ of refractive index can be resolved. As seen in the phase maps presented here, this type of variation is apparent in the fields around the human finger and can be extrapolated to be present around other living biological objects. Since we are interested in dynamic changes and not absolute values, we feel that this technique shows promise for tracking dynamic changes in thermal fields around biological objects.

The main limitation of the method used in this study is that the software in its current incarnation does not enable unwrapping the phase in time. This would enable tracking a specific air current over time and enable determination of the refractive index (or OPD changes) between frames of phase data. This type of calculation could be invaluable for a number of different applications such as modal analysis and mapping of air turbulence in a telescope dome. Dynamic interferometry is relatively new. The first dynamic interferometers were designed simply to get around vibration and air turbulence issues. As the field is evolving it is becoming apparent that dynamic interferometry has a huge advantage over standard phase measurement interferometry by being able to follow dynamic motions and capture dynamic events. A survey of vendors of dynamic interferometers indicates that they are in the process of incorporating this type of analysis into their products. It is anticipated that in the not too distant future dynamic analysis and visualization of motions and flows will be the industry standard.

\section{CONCLUSIONS}

Interferometry is sensitive enough to measure the difference in temperature gradients around objects of different temperatures. Adding the dynamic capability of short exposures capturing multiple interferograms simultaneously enables freezing effects of air currents in time. With the ability to capture movies of dynamic events, air currents, flows and dynamic motions can be tracked.

Our preliminary studies presented in this paper clearly show that it is possible to discern the difference between objects at different temperatures by looking at the gradient of the phase map around the object. This experiment has also shown that there is a difference in the dynamic air currents and temperature gradients around living biological objects and inanimate objects at the same temperature. Adding the dimension of time enables the study of subtle changes as a function of time. This type of measurement will enable the study of the dynamics of thermal emission from the human body. We anticipate that dynamic interferometry will enable the correlation of dynamic biofield measurements of thermal microbreezes to variations in metabolic function such as heart rate, respiration and EEG.

\section{ACKNOWLEDGEMENTS}

The authors wish to thank Steve Martinek and John Hayes of 4D Technology, Inc. for the use of their PhaseCam interferometer and Larry and Mark for developing the software "burst" mode for this study. We thank Prof. Art Gmitro for use of his Princeton Instruments camera. One of the authors (GES) is partially supported by NIH grant P20 AT00774 from the National Center for Complementary and Alternative Medicine (NCCAM). The contents of this paper are solely the responsibility of the authors and do not necessarily represent the official views of NCCAM or NIH. 


\section{REFERENCES}

1. B. Rubik, "The Biofield Hypothesis: Its Biophysical Basis and Role in Medicine," Journal of Alternative and Complementary Medicine 8(6), 703-717 (2002).

2. J. L. Oschman, Energy Medicine: The Scientific Basis (Churchill Livingstone, Edinburgh, 2000).

3. K. J. Hintz, G. L. Yount, I. Kadar, G. E. Schwartz, R. Hammerschlag, and S. Lin, "Bioenergy Definitions and Research Guidelines," Alternative Therapies in Health and Medicine 9(3 Suppl), 17A-34A (2003).

4. J. C. Wyant, "Dynamic Interferometry," Optics and Photonics News 14(4), 36-41 (2003).

5. J. C. Wyant, "Advances in Interferometric Metrology," in Proceedings of SPIE -- Volume 4927: Optical Design and Testing, Z. Weng, J. M. Sasian, and Y. Wang, eds. (SPIE, Bellingham, WA, 2002), pp. 154-162.

6. M. B. North-Morris, J. VanDelden, and J. C. Wyant, "Phase-Shifting Birefringent Scatterplate Interferometer," Applied Optics 41, 668-677 (2002).

7. C. L. Koliopoulos, "Simultaneous phase-shift interferometer," in Advanced Optical Manufacturing and Testing II, V. J. Doherty, ed. (SPIE, Bellingham, WA, 1992), pp. 119-127.

8. R. Smythe, et al., "Instantaneous Phase Measuring Interferometry," Optical Engineering 23(4), 361-364 (1984).

9. J. Hayes, "Dynamic interferometry handles vibration," Laser Focus World 38(3), 109-+ (2002).

10. James E. B. Millerd, Neil J.; Baer, James W.; Spuhler, Peter T., "Vibration insensitive, interferometric measurements of mirror surface figures under cryogenic conditions," in Proceedings of the SPIE, Volume 4842: Specialized Optical Developments in Astronomy, E. D. O. Atad-Ettedgui, Sandro, ed. (SPIE, Bellingham, WA, 2003), pp. 242-249.

11. J. E. Millerd, M. Schmucker, J. B. Hayes, R. Eng, J. Lassiter, H. P. Stahl, T. Rogers, J. B. Hadaway, and J. Geary, "Interferometric measurement of the vibrational characteristics of light-weight mirrors," in Proceedings of SPIE -- Volume 5180: Optical Manufacturing and Testing V, H. P. Stahl, ed. (SPIE, Bellingham, WA, 2004), pp. 211-218.

12. K. Creath and J. Schmit, "Phase-Measurement Interferometry," in Encyclopedia of Modern Optics, B. D. Guenther et. al, eds. (Academic Press, Dec 2004).

13. J. Hayes, "4D Technology Corporation Website", retrieved 1 May, 2004, http://www.4dtechnology.com/.

14. F. Bushroe, "Optical Device Engineering Corporation Website", retrieved 1 May, 2004, http://www.odecorp.com/.

15. C. L. Koliopoulos, "ADE Phase Shift Website", retrieved 1 May, 2004, http://www.phase-shift.com.

16. J. E. Millerd and N. J. Brock, "Method and apparatus for splitting, imaging, and measuring wavefronts in interferometry," US Patent 6,304,330 (16 Oct. 2001).

17. J. E. Millerd and N. J. Brock, "Methods and apparatus for splitting, imaging, and measuring wavefronts in interferometry," US Patent 6,552,808 (22 Apr. 2003).

18. K. Creath, "Phase-measurement interferometry techniques," in Progress in Optics, E. Wolf, ed. (Elsevier Science Publishers, Amsterdam, 1988), pp. 349-393.

19. D. W. Robinson, "Phase unwrapping methods," in Interferogram Analysis, D. W. Robinson and G. T. Reid, eds. (IOP Publishing, Bristol, 1993), pp. 194-229.

20. S. Cohen and F. A. Popp, "Biophoton emission of the human body," Journal of Photochemistry and Photobiology B-Biology 40(2), 187-189 (1997).

21. C. Choi, W. M. Woo, M. B. Lee, J. S. Yang, K. S. Soh, G. Yoon, M. Kim, C. Zaslawsky, and J. J. Chang, "Biophoton emission from the hands," Journal of the Korean Physical Society 41(2), 275-278 (2002).

22. K. Creath and G. E. Schwartz, "Biophoton Images of Plants: Revealing the Light Within," Journal of Alternative and Complementary Medicine 10(1), 23-26 (2004).

23. P. E. Ciddor, "Refractive index of air: new equations for the visible and near infrared," Applied Optics 35(9), 15661573 (1996). 